\title{
Prevalência do hipotiroidismo subclínico e repercussões sobre 0 perfil lipídico e massa óssea em mulheres na pós-menopausa
}

\author{
Prevalence of subclinical hypothyroidism and its effects on lipidic profile and bone mineral \\ density in postmenopausal women
}

Eliana Aguiar Petri Nahas ${ }^{1}$, Jorge Nahas-Neto², Paulo Eduardo Mattos Ferreira Santos ${ }^{3}$, Gláucia Maria Ferreira da Silva Mazeto ${ }^{4}$, Ivete Dalben ${ }^{5}$, Anaglória Pontes ${ }^{6}$, Paulo Traiman ${ }^{7}$

\section{RESUM0}

Objetivo: avaliar a prevalência do hipotiroidismo subclínico e suas repercussões sobre o perfil lipídico e a densidade mineral óssea (DMO) em mulheres na pós-menopausa. Métodos: trata-se de estudo transversal com recuperação de dados de prontuários de pacientes acompanhadas em ambulatório de climatério. Critérios de inclusão: mulheres na pós-menopausa com dosagem do hormônio estimulador da tiróide (TSH) e de tiroxina livre ( $\left.\mathrm{T}_{4}-\mathrm{L}\right)$. Critérios de exclusão: hipertiroidismo e carcinoma de tiróide. Considerou-se hipotiroidismo subclínico valores de TSH superiores a 5,0 mUI/mL e T ${ }_{4}-\mathrm{L}_{\text {normal. Foram }}$ selecionadas 320 pacientes (idade 55,2 $\pm 6,4$ anos) divididas em 3 grupos: função tiroideana normal ( $\mathrm{n}=208$ ), hipotiroidismo subclínico (n=53) e hipotiroidismo clínico sob tratamento $(\mathrm{n}=59)$. Foram analisados dados clínicos, uso de terapia hormonal, índice de massa corpórea (IMC=kg/ $\mathrm{m}^{2}$ ), perfil lipídico (colesterol total, HDL, LDL, triglicerídeos) e DMO da coluna lombar e fêmur. Na análise estatística, as diferenças entre as médias dos grupos foram comparadas utilizando-se a análise de variância (ANOVA). Para múltipla comparação, assumindo que a variância era diferente entre os grupos, utilizou-se o método de Tukey. Resultados: o hipotiroidismo subclínico foi diagnosticado em 16,1\% dos casos. Os grupos foram homogêneos quanto às características clínicas, IMC e perfil lipídico e uso de terapêutica hormonal. Nas pacientes com hipotiroidismo subclínico ou clínico encontrou-se menor frequêência de osteopenia na coluna lombar e fêmur quando comparadas às eutiroidianas $(\mathrm{p}<0,001)$. Houve correlação negativa entre os valores de TSH e DMO da coluna lombar e fêmur $(\mathrm{p}<0,001)$. Não se constatou correlação entre os valores de TSH e idade, tempo de menopausa, IMC e perfil lipídico. O total de usuárias de

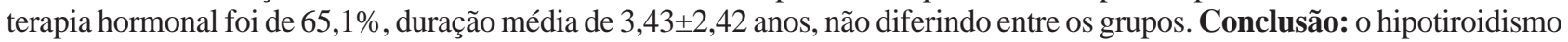
subclínico com prevalência de 16,1\% na pós-menopausa associou-se à baixa DMO, mas sem repercussões sobre o perfil lipídico.

PALAVRAS-CHAVE: Hipotiroidismo; Pós-menopausa; Densidade óssea; Lipides

\section{ABSTRACT}

Purpose: to evaluate the prevalence of subclinical hypothyroidism and its effects on lipidic profile and bone mineral density (BMD) in postmenopausal women. Methods: a cross-sectional study with survey of data from medical records of patients attended at a climacteric outpatient clinic. Inclusion criteria: postmenopausal women with measured thyroid-stimulating hormone (TSH) and free thyroxin ( $\mathrm{T}_{4}-\mathrm{L}$ ). Exclusion criteria: hyperthyroidism and thyroid cancer. Values of TSH $>5.0 \mathrm{mIU} / \mathrm{mL}$ and normal $\mathrm{T}_{4}$ - $\mathrm{L}$ were considered to be subclinical hypothyroidism. The 329 selected women (55.2 \pm 6.4 years) were divided into three groups: normal thyroid function (control) $(n=208)$, subclinical hypothyroidism $(n=53)$ and clinical hypothyroidism under treatment $(\mathrm{n}=59)$. Clinical data, hormonal therapy use, body mass index $\left(\mathrm{BMI}=\mathrm{kg} / \mathrm{m}^{2}\right)$, lipid profile (total cholesterol, HDL, LDL, triglycerides) and BMD of lumbar column and femur were obtained. Results: subclinical hypothyroidism was

Faculdade de Medicina de Botucatu - Universidade Estadual Paulista - UNESP - Botucatu (SP), Brasil.

1 Professora Doutora do Departamento de Ginecologia e Obstetrícia - Universidade Estadual Paulista - UNESP - Botucatu (SP), Brasil.

2 Professor Doutor do Departamento de Ginecologia e Obstetrícia - Universidade Estadual Paulista - UNESP - Botucatu (SP), Brasil.

3 Bolsista do Programa Institucional de Bolsa de Iniciação Científica da Universidade Estadual Paulista - UNESP - Botucatu (SP), Brasil.

4 Professora Doutora do Departamento de Clínica Médica - Universidade Estadual Paulista - UNESP - Botucatu (SP), Brasil.

5 Professora Doutora do Departamento de Saúde Pública - Universidade Estadual Paulista - UNESP - Botucatu (SP), Brasil.

6 Professora Doutora do Departamento de Ginecologia e Obstetrícia - Universidade Estadual Paulista - UNESP - Botucatu (SP), Brasil.

7 Professor Livre Docente do Departamento de Ginecologia e Obstetrícia - Universidade Estadual Paulista - UNESP - Botucatu (SP), Brasil.

Correspondência: Eliana Aguiar Petri Nahas

Departamento de Ginecologia e Obstetrícia

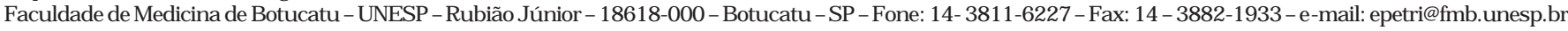


diagnosed in $16.1 \%$ of the cases. The groups were homogeneous regarding clinical features, BMI or lipidic profile. BMD in lumbar column and femur was lower in subclinical and clinical hypothyroidism than in euthyroidism $(\mathrm{p}<0.001)$. There was a negative correlation between values of TSH and BMD of lumbar column and femur $(\mathrm{p}<0.001)$. There was no correlation between TSH values and age, menopause time, BMI, and lipid profile. The total of hormonal therapy users was $65.1 \%$, mean duration of $3.43 \pm 2.42$ years, not differing between the groups. Conclusion: subclinical hypothyroidism with prevalence of $16.1 \%$ in postmenopausal women was associated with lower BMD, with no effects on lipid profile.

KEYWORDS: Hypothyroidism; Postmenopause; Bone density; Lipids

\section{Introdução}

O hipotiroidismo subclínico é definido como elevação dos níveis séricos do hormônio estimulador da tiróide (TSH), tendo a tiroxina livre $\left(\mathrm{T}_{4}-\mathrm{L}\right)$ e a triiodotironina, dentro da taxa de normalidade. Sinais clínicos ou história de doença tiroidiana estão ausentes ${ }^{1,2}$. A prevalência desta condição na população adulta americana é de 4 a $10 \%{ }^{3}$. Entretanto, em mulheres com mais de 60 anos está presente em até $20 \%{ }^{3}$. O diagnóstico é laboratorial, pela dosagem de TSH, que demonstra alta sensibilidade (98\%) e especificidade (92\%) ${ }^{2}$. As manifestações clínicas do hipotiroidismo, a princípio, são inaparentes, graduais e freqüentemente atribuídas ao envelhecimento e ao climatério $^{4}$. Se nenhuma rotina laboratorial é realizada, muitas mulheres afetadas permanecem sem diagnóstico. Em recente estudo, no qual se realizaram dosagens de $\mathrm{TSH}$ e $\mathrm{T}_{4}-\mathrm{L}$ em 300 pacientes internadas em hospital geral, constatouse que $12,6 \%$ apresentavam disfunções tiroidianas não suspeitadas na internação, entre estas, o hipotiroidismo subclínico em $28,9 \%$ dos casos ${ }^{5}$.

A alta incidência de doenças tiroidianas não reconhecidas clinicamente justifica avaliações laboratoriais rotineiras ${ }^{4,5}$. Recomenda-se que sejam realizadas dosagens de TSH a cada cinco anos, iniciando aos 35 anos de idade ${ }^{1}$. O rastreamento está indicado principalmente em mulheres na pósmenopausa, de acordo com sinais e sintomas potencialmente atribuídos às disfunções tiroidianas $^{3}$. O hipotiroidismo subclínico é freqüente na pós-menopausa, associando-se a efeitos adversos à saúde da mulher que podem ser evitados pela simples dosagem do $\mathrm{TSH}^{2,4}$.

A integridade do aparelho ósseo da mulher na pós-menopausa depende não só de sua condição estrogênica, mas também de outros fatores hormonais. A tiróide, pela ação direta de seus hormônios sobre o metabolismo ósseo, tem papel importante no processo de remodelação óssea ${ }^{6}$. A elevação dos hormônios tiroidianos estimula a reabsorção óssea, com perda de massa óssea ${ }^{6}$. Mulheres na pós-menopausa com hipotiroidismo subclínico, usuárias de hormônio tiroidiano (levotiroxina), apresentam menores valores de densidade mineral óssea (DMO) quando comparadas a mulheres eutiroidianas ${ }^{7}$. A reposição de hormônios tiroidianos em pacientes com hipotiroidismo subclínico poderia interferir negativamente sobre a remodelação óssea e a $\mathrm{DMO}^{8}$.

A relação entre o hipotiroidismo subclínico e o risco cardiovascular é complexa ${ }^{9}$. Observa-se maior prevalência de aterosclerose aórtica e infarto do miocárdio em mulheres na pós-menopausa com hipotiroidismo subclínico quando comparadas às eutiroidianas, de mesma faixa etária ${ }^{10}$. Situações de risco para doença coronariana como alterações no perfil lipídico, com hipercolesterolemia e hipertrigliceridemia, são freqüentemente observadas no hipotiroidismo subclínico ${ }^{9,11}$. Em recente meta-análise de 195 estudos sobre hipotiroidismo subclínico, encontraram-se evidências relacionando elevados valores de TSH com aumento do colesterol total ${ }^{3}$.

Baseados nestes dados, o objetivo deste estudo foi avaliar a prevalência do hipotiroidismo subclínico e suas repercussões sobre o perfil lipídico e a DMO em mulheres na pós-menopausa, atendidas em um ambulatório de climatério.

\section{Pacientes e Métodos}

Foi conduzido estudo de corte transversal, por meio do levantamento de dados de prontuários. O grupo populacional foi constituído de 320 pacientes, com idade entre 44 a 75 anos, acompanhadas em ambulatório de climatério, de fevereiro a dezembro de 2003. Os critérios de inclusão foram: menopausadas (data da última menstruação há pelo menos 12 meses), avaliação laboratorial incluindo dosagem do $\mathrm{TSH}_{1} \mathrm{~T}_{4}$-L e perfil lipídico, e com mensuração da DMO prévia. Os critérios de exclusão foram diagnósticos de hipertiroidismo e carcinoma de tiróide. O projeto de pesquisa recebeu parecer favorável do Comitê de Ética em Pesquisa da Faculdade de Medicina de Botucatu - UNESP (Of. 117/2003).

Todas as pacientes atendidas no ambulatório submeteram-se rotineiramente a anamnese 
detalhada, exame físico geral e ginecológico. Baseado nas informações dos prontuários, foi preenchido o protocolo para obtenção dos seguintes dados: idade, idade da menarca, paridade, idade e tempo de menopausa, histórico de disfunções tiroidianas, uso ou não de terapia hormonal $(\mathrm{TH})$, peso e estatura. O índice de massa corpórea (IMC) foi calculado dividindo-se o peso pelo quadrado da altura. Seguindo os critérios da Organização Mundial da Saúde ${ }^{12}$, foi considerado IMC menor que 18,5 como baixo peso, de 18,5 a 24,9 normal, de 25 a 29,9 pré-obesidade, de 30 a 34,9 obesidade classe I, de 35 a 39,9 obesidade classe II e maior ou igual a 40 obesidade classe III.

Classificou-se como portadora de hipotiroidismo subclínico a paciente que apresentasse valores de TSH superiores a $5 \mathrm{mIU} / \mathrm{mL}$, com niveis normais de tiroxina livre $\mathrm{T}_{4}-\mathrm{L}$, baseado nos critérios de normalidade segundo os métodos empregados. $\mathrm{O}$ TSH e o $\mathrm{T}_{4}-\mathrm{L}$ foram quantificados pelo Sistema Immulite ${ }^{\circledR}\left(\mathrm{DPC}^{\circledR}\right.$, USA), que emprega imunoensaio por quimioluminescência, em fase sólida, para uso em analisador automático, designado para leitura quantitativa hormonal. A sensibilidade analítica para TSH foi de $0,002 \mathrm{mIU} / \mathrm{mL}$ e para T4-L, de 0,15 ng/dL. Os coeficientes de variação intra-ensaio e interensaio foram, respectivamente, para TSH de 7,0 e $11 \%$ e para $\mathrm{T}_{4}-\mathrm{L}$, de $7,2 \%$. As taxas de normalidade para os exames são: TSH de 0,49 a 4,67 mIU/mL e T $-\mathrm{L}$ de 0,71 a $1,85 \mathrm{ng} / \mathrm{dL}$.

Para a análise do perfil lipídico foram obtidos os valores do colesterol total (CT), HDL, LDL e triglicerídeos (TG). O CT, o HDL e o TG foram quantificados por método colorimétrico, utilizando-se reagentes comerciais específicos (Sera-Pak ${ }^{\circledR}$, Bayer, USA), para uso em analisador bioquímico automático. Calculou-se o LDL pela fórmula de Friedewald, subtraindo-se o valor do CT, da soma do HDL e do TG dividido por cinco. As taxas de normalidade são: CT inferior a $200 \mathrm{mg} / \mathrm{dL}$, HDL superior a $35 \mathrm{mg} / \mathrm{dL}$, LDL inferior a $130 \mathrm{mg} / \mathrm{dL}$ e TG inferior a $150 \mathrm{mg} / \mathrm{dL}$.

Foram utilizados os resultados da avaliação da DMO obtidos pela absorciometria de dupla emissão (Dexa) (Sophos ${ }^{\circledR}$, L-XRA, França), na coluna lombar (L1 a L4) e colo femural. O Dexa usa como fonte o raios-X e mede a DMO em g/ $\mathrm{cm}^{2}$, que é expressa em unidades de desvio-padrão (DP). Na classificação dos resultados empregaram-se os critérios da Organização Mundial da Saúde ${ }^{13}$, que utiliza como faixa de referência a DMO do adulto jovem. Classificou-se DMO como normal quando Tscore fosse superior a -1,0 DP da faixa de referência, osteopenia entre - 1,0 a -2,5 DP e osteoporose inferior a -2,5 DP. O coeficiente de precisão desse método, em mulheres na pós-menopausa, é de $2 \%$.

$\mathrm{Na}$ análise estatística, as diferenças entre as médias dos grupos foram comparadas utilizando-se a análise de variância (ANOVA). Para múltipla comparação, assumindo que a variância era diferente entre os grupos, utilizou-se o método de Tukey. Para verificar a diferença de freqüência entre os grupos, quanto ao uso de terapia hormonal, empregou-se o teste do $\chi^{2}$. Na comparação entre os grupos, as diferenças entre as medianas da DMO da coluna e fêmur foram estabelecidas pelo teste não paramétrico de Kruskal-Wallis, para amostras independentes. A correlação entre pares de variáveis quantitativas foi obtida pelo coeficiente de correlação de Pearson. Os testes estatísticos foram bicaudais, com o nível de significância escolhido de 5\%. Os dados foram calculados com o auxílio do software SPSS, versão 10.0.5.

\section{Resultados}

As pacientes selecionadas foram divididas em três grupos: com função tiroidiana normal (eutiroidianas) $(\mathrm{n}=208)$, com hipotiroidismo subclínico $(n=53)$ e com hipotiroidismo clínico, sob tratamento com levotiroxina $(n=59)$. A prevalência do hipotiroidismo subclínico foi de $16,1 \%$. As características clínicas das pacientes dos três grupos foram submetidas à comparação estatística e estão representadas na Tabela 1. Verificou-se que os grupos foram homogêneos paras as seguintes variáveis: idade, idade da menarca, idade da menopausa, paridade e IMC. Os valores médios do IMC foram compativeis com pré-obesidade ou sobrepeso (de 25 a 29,9 kg/m²), nos três grupos (Tabela 1).

Tabela 1 - Comparação das características clínicas entre os grupos (valores médios \pm desvio-padrão).

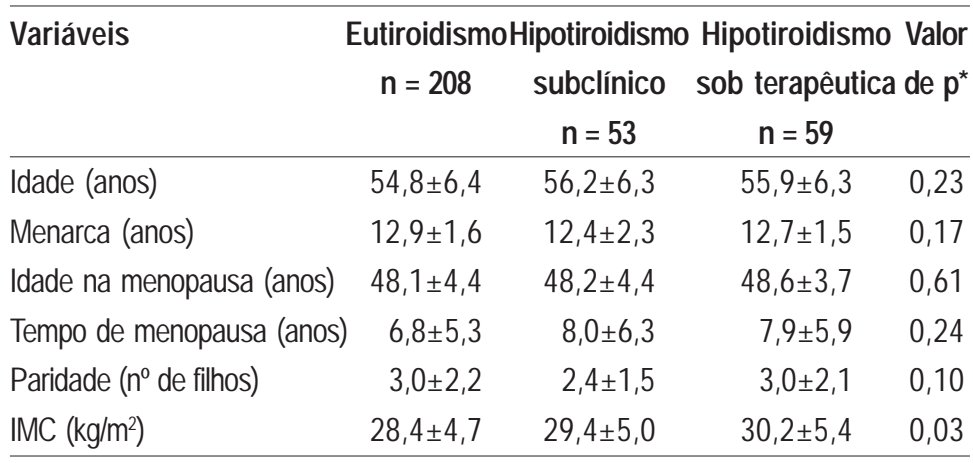

*Diferença significativa entre grupos se $p<0,05$ (ANOVA).

IMC = índice de massa corpórea. 
Confrontando-se os valores de CT, HDL, LDL e TG não se observou diferença entre os grupos (Tabela 2). Baseado nos critérios para a divisão dos grupos, as pacientes com hipotiroidismo subclínico apresentaram maiores valores médios de TSH $(7,9 \pm 3,2 \mathrm{mUI} / \mathrm{mL})$ e menores de $\mathrm{T}_{4}-\mathrm{L}(1,0 \pm 0,3 \mathrm{ng} /$ $\mathrm{dL})$ quando comparadas as eutiroidianas $(2,0 \pm 1,3$ $\mathrm{mUI} / \mathrm{mL}$ e $1,3 \pm 0,3 \mathrm{ng} / \mathrm{dL}$ ) e com hipotiroidismo clínico em tratamento $(3,1 \pm 2,0 \mathrm{mUI} / \mathrm{mL}$ e $1,3 \pm 0,3 \mathrm{ng} /$ dL, respectivamente) (Tabela 2 ).

Tabela 2 - Comparação entre os valores laboratoriais para avaliação tireoidiana e perfil lipídico entre os grupos (valores médios \pm desvio-padrão).

\begin{tabular}{|c|c|c|c|c|}
\hline Variáveis & $\begin{array}{c}\text { Eutiroidismo } \\
\qquad n=208\end{array}$ & $\begin{array}{c}\text { lipotiroidismo } \\
\text { subclínico } \\
n=53\end{array}$ & $\begin{array}{l}\text { Hipotiroidismo } \\
\text { sob terapêutic } \\
n=59\end{array}$ & $\begin{array}{l}\text { o Valor } \\
\text { cade p* }\end{array}$ \\
\hline TSH (mUl/mL) & & & & $<0,001$ \\
\hline $\mathrm{T}_{4}$ livre (ng/dL) & $1,0 \_0,0$ & $1,0 \pm 0,3$ & $1,3 \pm 0,3$ & $<0,00$ \\
\hline Colesterol total (mg/dL) & $219,1 \pm 40,4$ & $223,8 \pm 40,3$ & $218,7 \pm 39,4$ & 0,72 \\
\hline $\mathrm{HDL}(\mathrm{mg} / \mathrm{dL})$ & $53,5 \pm 13,3$ & & $53,7 \pm 15,0$ & 0,73 \\
\hline LDL (mg/dL) & $133,6 \pm 36,7$ & $139,0 \pm 38,3$ & $133,4 \pm 37,2$ & 0,62 \\
\hline Triglicerídeos (mg/dL) & $147,8 \pm 70,0$ & $155,7 \pm 72,5$ & $149,6 \pm 69,5$ & 0,77 \\
\hline
\end{tabular}

*Diferença significativa entre grupos se $p<0,05$ (ANOVA).

Nas pacientes com hipotiroidismo subclínico e naquelas com hipotiroidismo prévio sob terapêutica, observaram-se valores medianos de DMO compativeis com osteopenia (T-score de 1,0 a -2,5 DP), na coluna lombar e fêmur. Nas pacientes com função tiroidiana normal, os valores medianos da DMO encontravam-se dentro da normalidade (T-score $>-1,0 \mathrm{DP})(\mathrm{p}<0,05)$ (Tabela 3 ). Houve correlação negativa entre os valores de TSH e a DMO da coluna lombar e do fêmur $(\mathrm{p}<0,001)$. Não se observou correlação entre os valores de TSH e a idade, o tempo de menopausa, o IMC e o perfil lipídico.

Tabela 3 - Comparação dos valores da densidade mineral óssea entre os grupos (valores expressos em mediana, com os valores mínimo e máximo entre parênteses).

\begin{tabular}{lcccc}
\hline Variáveis & Eutiroidismo Hipotiroidismo Hipotiroidismo & Valor \\
subclínico & & sob terapêutica & de $\mathbf{p}^{*}$ \\
& $\mathbf{n}=\mathbf{2 0 8}$ & $\mathbf{n}=\mathbf{5 3}$ & $\mathbf{n = 5 9}$ & \\
\hline DMO coluna & $-0,9$ & $-2,0$ & $-1,8$ & $<0,001$ \\
& $(-3,1$ e 2,6$)$ & $(-3,6$ e $-0,4)$ & $(-4,3$ e 0,4$)$ & \\
DMO fêmur & $-0,8$ & $-1,7$ & $-1,3$ & $<0,001$ \\
& $(-3,6$ e 2,7$)$ & $(-3,8$ e $-0,1)$ & $(-3,6$ e 0,4$)$ & \\
\hline
\end{tabular}

*Diferença significativa entre grupos se $p<0,05$ (teste de Kruskal-Wallis). $\mathrm{DMO}=$ densidade mineral óssea.

Com relação o uso de $\mathrm{TH}$, constatou-se que $65,6 \%(210 / 320)$ eram usuárias, com duração média de $3,4 \pm 2,4$ anos, e $34,4 \%(110 / 320)$ não usuárias, não havendo diferenças entre os grupos. Entre os esquemas de TH, 51,9\% (109/210) utilizavam a associação de estrogênio e progestagênio em esquema combinado-contínuo, $22,4 \%(47 / 210)$ estrogenioterapia isolada, $16,2 \%$ (34/210) em seqüencial-cíclico e 9,5\% (20/210) fitoestrogênios.

\section{Discussão}

A freqüência de hipotiroidismo subclínico caracteristicamente aumenta com a idade, particularmente na mulher após a quinta década de vida, com prevalência de aproximadamente $20 \%{ }^{3,4}$. Aproximadamente 2 a $5 \%$ dos casos progridem para hipotiroidismo clínico dentro do primeiro $\mathrm{ano}^{3,9}$. Decorre freqüentemente da falência primária da tiróide, em virtude de processo auto-imune de destruição do órgão ${ }^{3}$ O rastreamento da disfunção tiroidiana, com dosagens séricas de TSH, é recomendado para mulheres na pós-menopausa ${ }^{1,4}$. Nesta casuística, a avaliação da função tiroidiana, introduzida na rotina do atendimento de mulheres na pós-menopausa em ambulatório de climatério, constatou a presença de hipotiroidismo subclínico em 16,1\%.

$\mathrm{Na}$ presente pesquisa, as pacientes com hipotiroidismo subclínico, assim como aquelas com hipotiroidismo clínico sob tratamento, apresentaram menores valores médios de DMO na coluna lombar e fêmur, classificada pela densitometria como osteopenia. Houve correlação negativa entre os valores de TSH e a DMO. Por outro lado, nas pacientes com função tiroidiana normal, a DMO encontrava-se dentro da normalidade. Pacientes com hipotiroidismo, sob terapêutica com levotiroxina, têm elevado risco de osteoporose, pois cursam com algum grau de tirotoxicose subclínica medicamentosa ${ }^{14,15}$. O aumento da tiroxina estimula os osteoclastos, elevando a reabsorção óssea, com perda de osso cortical e trabecular ${ }^{15}$. Nas pacientes com hipotiroidismo subclínico, o tratamento é controverso pela possibilidade de que a reposição de hormônios tiroidianos interfira negativamente sobre o metabolismo ósseo, elevando o risco de osteoporose $\mathrm{e}^{4,9,14}$. Em estudo no qual se avaliou a massa óssea de mulheres com hipotiroidismo subclínico na pós-menopausa, as pacientes foram submetidas a TH isolada ou associada a levotiroxina ou ficaram sem tratamento. Após três anos de seguimento, constatou-se que as pacientes sob TH associada a levotiroxina e aquelas sem tratamento apresentaram redução na DMO quando comparadas ao grupo sob TH isolado. Os 
autores concluem que a levotiroxina pode afetar os efeitos benéficos da estrogenioterapia sob o ganho de massa óssea em mulheres na pós-menopausa ${ }^{7}$. Na presente pesquisa, as pacientes com hipotiroidismo prévio e DMO diminuída estavam em tratamento com levotiroxina. Não foram encontrados estudos correlacionando o hipotiroidismo subclínico não tratado com redução da massa óssea.

Neste levantamento não foram constatadas diferenças nos valores de CT, LDL, HDL e TG entre as pacientes eutiroidianas, com hipotiroidismo sob terapêutica e subclínico. Do mesmo modo, Vierhapper et al. ${ }^{16}$, estudando 4.886 pacientes eutiroideanas e $1.055 \mathrm{com}$ hipotiroidismo subclínico, não demonstraram correlação entre os valores de TSH e de LDL. As pacientes com hipotiroidismo subclínico acompanhado de hipercolesterolemia são consideradas de maior risco cardiovascular ${ }^{9,10}$. Estudos demonstraram que pacientes com TSH acima de 5,0 mUI/mL têm significativamente maiores valores de colesterol total e LDL quando comparadas a eutiroidianas ${ }^{11,17,18}$. Em revisão de literatura, se observou redução média de 5\% nos valores de CT e LDL em mulheres com hipotiroidismo subclínico, tratadas com levotiroxina, principalmente naquelas com elevados valores pré-tratamento ${ }^{19}$. Essas observações sugerem que pacientes com hipotiroidismo subclíncio, com hipercolesterolemia, teriam benefícios com a reposição de hormônios tiroidianos. Todavia, questiona-se a efetividade da terapêutica com levotiroxina no hipotiroidismo subclínico, para modificar os riscos cardiovasculares ${ }^{3}$.

Existem controvérsias com relação ao significado clínico do hipotiroidismo subclínico e suas repercussões sobre a saúde da mulher na pósmenopausa. Os benefícios em detectar e tratar não estão bem estabelecidos quando TSH está entre 5 e $10 \mathrm{mUI} / \mathrm{mL}$, entretanto pode ser benéfico se os valores de TSH encontram-se acima de $10 \mathrm{mUI} /$ $\mathrm{mL}^{20}$. Em estudo de revisão, Tamilia e Silva ${ }^{21}$ concluem que no hipotiroidismo subclínico a terapêutica seria aconselhável para pacientes com TSH maior que $10 \mathrm{mUI} / \mathrm{mL}$ e naquelas com TSH entre 5 e $9 \mathrm{mUI} / \mathrm{mL}$ se houver perfil lipídico aterogênico ou doença coronariana. O tratamento com levotiroxina é simples e relativamente barato. Entretanto, a administração difere substancialmente do $\mathrm{T}_{4}$ endógeno, pois é menos ativo, não tem a atividade intrínseca hormonal e tem efeito lipogênico sobre o fígado. Assim, a terapia é justificável quando se evidenciam benefícios para a paciente ${ }^{21}$.

Desde que as pacientes com disfunções tiroidianas estejam controladas, não há contra- indicações para a $\mathrm{TH}^{20}$. Estima-se que aproximadamente 5\% das mulheres na pós-menopausa sejam tratadas com estrogenioterapia e hormônios tiroidianos ${ }^{22}$. Pelo efeito da primeira passagem hepática, o estrogênio por via oral eleva as concentrações da globulina carreadora da tiroxina (TBG), diminuindo a fração bioativa da tiroxina circulante $^{23}$. Em 2001, Arafah ${ }^{24}$, avaliando 250 mulheres na pós-menopausa, com hipotiroidismo em uso de levotiroxina, constatou que necessitam de ajustes na posologia após início da TH. Recomenda avaliação da função da tiróide após 12 semanas de TH em mulheres sob tratamento com hormônio tiroidiano.

As doenças da tiróide, particularmente o hipotiroidismo, são freqüentes na pós-menopausa. Os ginecologistas preocupados com a saúde da mulher devem estar familiarizados com a multiplicidade de sinais e sintomas das disfunções tiroidianas. A disponibilidade da dosagem de TSH na rotina do atendimento torna o diagnóstico direto e barato. $\mathrm{O}$ tratamento segue princípios bem estabelecidos. Alguns ginecologistas com formação nessa área poderão executar o tratamento inicial, outros encaminharão as pacientes ao endocrinologista ${ }^{25}$. Na presente casuística, o hipotiroidismo subclínico, com prevalência de $16,1 \%$ em mulheres na pós-menopausa, associouse a menor DMO, sem repercussões sobre o perfil lipídico.

\section{Referências}

1. Ladenson PW, Singer PA, Ain KB, Bagchi N, Bigos ST, Levy EG, et al. American Thyroid Association guidelines for detection of thyroid dysfunction. Arch Intern Med. 2000;160(11):1573-5.

2. U.S. Preventive Services Task Force. Screening for thyroid disease: recommendation statement. Ann Intern Med. 2004;140(2):125-7.

3. Surks MI, Ortiz E, Daniels GH, Sawin CT, Col NF, Cobin RH, et al. Subclinical thyroid disease: scientific review and guidelines for diagnosis and management. JAMA. 2004;291(2):228-38.

4. Schindler AE. Thyroid function and postmenopause. Gynecol Endocrinol. 2003;17(1):79-85.

5. Torre R, Del Monte P, Bernasconi D, Marugo A, Poggi $\mathrm{P}$, Leoncini R, et al. Screening for thyroid disorders in elderly patients. Recenti Prog Med. 2004;95(6):308-11.

6. Greenspan SL, Greenspan FS. The effect of thyroid hormone on skeletal integrity. Ann Intern Med. 1999;130(9):750-8. 
7. Pines A, Dotan I, Tabori U, Villa Y, Mijatovic V, Levo $\mathrm{Y}$, et al. L-thyroxine prevents the bone-conserving effect of HRT in postmenopausal women with subclinical hypothyroidism. Gynecol Endocrinol. 1999;13(3):196-201.

8. Meier C, Beat M, Guglielmetti M, Christ-Crain M, Staub JJ, Kraenzlin M. Restoration of euthyroidism accelerates bone turnover in patients with subclincial hypothyroidism; a randomized controlled trial. Osteoporos Int. 2004;15(3):209-16.

9. Palmieri EA, Fazio S, Lombardi G, Biondi B. Subclinical hypothyroidism and cardiovascular risk: a reason to treat? Treat Endocrinol. 2004;3(4):23344.

10. Hak AE, Pols HA, Visser TJ, Drexhage HA, Hofman A, Witteman JC. Subclinical hypothyroidism is an independent risk factor for atherosclerosis and myocardial infarction in elderly women: the Rotterdam Study. Ann Intern Med. 2000;132(4):2708.

11. Cabral MD, Costa AJL, Santos M, Vaisman M. Lipid profile alterations in subclinical hypothyroidism. Endocrinologist. 2004; 14(3):121-5.

12.WHO Study Group. Obesity: preventing and managing the global epidemic. Report of a WHO consultation on obesity. Geneva: WHO; 1997.

13. WHO Study Group. Assessment of fracture risk and its application to screening for postmenopausal osteporosis. Geneva: WHO; 1994.

14. Hadji P, Hars O, Sturm G, Bauer T, Emons G, Schultz KD. The effect of long-term, non-suppressive levothyroxine treatment on quantitative ultrasonometry of bone in women. Eur J Endocrinol. 2000;142(5):445-50.

15. Chen CH, Chen JF, Yang BY, Liu RT, Tung SC, Chien $\mathrm{WY}$, et al. Bone mineral density in women receiving thyroxine suppressive therapy for differentiated thyroid. J Formos Med Assoc. 2004;103(6):442-7.
16. Vierhapper H, Nardi A, Grosser P, Raber W, Gessl A. Low-density lipoprotein cholesterol in subclinical hypothyroidism. Thyroid. 2000;10(11):981-4.

17. Canaris GJ, Manowitz NR, Mayor G, Ridgway EC. The Colorado thyroid disease prevalence study. Arch Intern Med. 2000;160(4):526-34.

18.Hueston WJ, Pearson WS. Subclinical hypothyroidism and the risk of hypercholesterolemia. Ann Fam Med. 2004;2(4)3515.

19. Danese MD, Ladenson PW, Meinert CL, Powe NR. Clinical review: effect of thyroxine therapy on serum lipoproteins in patients with mild thyroid failure: a quantitative review of the literature. J Clin Endocrinol Metab. 2000;85(9):2993-3001.

20. Col NF, Surks MI, Daniels GH. Subclinical thyroid disease: clinical applications. JAMA. 2004;291(2):239-43.

21. Tamilia M, Silva JE. Subclinical hypothyroidism: to treat or not to treat? Curr Opin Endocrinol Diabetes. 2001;8(5):247-52.

22. Mazer NA. Interaction of estrogen therapy and thyroid hormone replacement in postmenopausal women. Thyroid. 2004; 14 Suppl 1:S27-34.

23. Marqusee E, Braverman LE, Lawrence JE, Carroll JS, Seely EW. The effect of droloxifene and estrogen on thyroid function in postmenopausal women. J Clin Endocrinol Metab. 2000;85(11):4407-10.

24. Arafah BM. Increased need for thyroxine in women with hypothyroidism during estrogen therapy. N Engl J Med. 2001;344(23):1743-9.

25. Redmond GP. Thyroid dysfunction and women's reproductive health. Thyroid. 2004;14 Suppl 1:S515. 